\title{
The Negative Binomial Distribution as a Renewal Model for the Recurrence of Large Earthquakes
}

\author{
Alejandro Tejedor (1), Javier B. Gómez (2) and Amalio F. Pacheco (3)
}

(1) Saint Anthony Falls Laboratory, Department of Civil Engineering, University of Minnesota, 2 Third Avenue SE, Minneapolis, Mn 55455; (2) Department of Earth Sciences, University of Zaragoza, 50009 Zaragoza, Spain; (3) Department of Theoretical Physics, University of Zaragoza, 50009 Zaragoza, Spain

Abbreviated title: The NBD as a renewal model for earthquakes

\section{ACCEPTED MANUSCRIPT}

Pure and Applied Geophysics, vol. 172(1), pp. 23-31

Published online 26 June 2014 


\begin{abstract}
The Negative Binomial Distribution is presented as the waiting time distribution of a cyclic Markov model. This cycle simulates the seismic cycle in a fault. As an example, this model, that can describe recurrences with aperiodicities between 0 and 0.5 , is used to fit the Parkfield, California, earthquake series in the San Andreas Fault. The performance of the model in the forecasting is expressed in terms of error diagrams and compared with other recurrence models from literature.
\end{abstract}

Key words: negative binomial distribution; renewal process; seismic cycle; earthquake forecasting

\title{
1. Introduction
}

The elastic-rebound model is the canonical "macroscopic" theory of great earthquakes (REID, 1910, SCHOLZ, 2002). It states that a great earthquake will occur where large elastic strains have accumulated in the crust. The earthquake itself will relieve most of the strain which will then accumulate slowly again by a steady input of tectonic stress until the elastic strain becomes sufficiently large for another earthquake to ensue. The duration of this earthquake cycle (the time between two consecutive large earthquakes) is the ratio of the strain released during an earthquake to the rate of input of tectonic strain by plate motion.

Because the Earth's crust is heterogeneous and faults are not isolated from each other, the earthquake cycle of a specific fault is not periodic. So, although the elastic-rebound model is in essence a deterministic theory, its application to a heterogeneous and interacting crust implies its translation into a probabilistic framework.

The variability of the duration of a cycle (either real earthquakes on a fault or synthetic earthquakes in a model) can be appropriately defined in the context of a probability density function (pdf) by means of the coefficient of variation, $\alpha$, the ratio of the standard deviation $\sigma$ to the mean $\mu$ of the pdf: 


$$
\alpha=\frac{\sigma}{\mu}
$$

In the seismological literature the coefficient of variation is also known as the aperiodicity, a very descriptive name when applied to the duration of the earthquake cycle: $\alpha=0$ gives perfectly periodic cycles, $0<\alpha<1$ quasiperiodic cycles, and $\alpha>1$ clustering of events. The case $\alpha=1$ is particularly important because the exponential distribution has this property, and the exponential distribution is the pdf of an earthquake cycle where large earthquakes occur in time following a Poisson distribution (i.e, they are random in time). In actual seismic faults the aperiodicity of the earthquake series is always less than one (SYKES AND MENKE, 2006, ELLSWORTH et al, 1999, ABAIMOV et al, 2007).

RIKITAKE (1974) was the first to formally introduce a probabilistic description of the occurrence times of specific earthquakes. He treated earthquake recurrence as a renewal process, in which the times between successive events (in this case the large earthquakes in a specific fault) are assumed to be independent and independently distributed random variables.

Since then several authors have proposed probabilistic versions of the elastic-rebound model in the shape of a plethora of probability distribution functions (pdfs) for the duration of the earthquake cycle: exponential (UTSU, 1984; SORNETTE and KNOPOFF, 1997; MATTHEWS et al., 2002), Weibull (UtSU, 1984; SORNETte and KNOPOFF, 1997; MAtTheWS et al., 2002; FerRÁEs, 2003; GÓMEZ and PACHeCO, 2004; ABAimOV et al., 2007, 2008; GolZ et al., 2009), log-normal (UTSU, 1984; SORNETTE and KNOPOFF, 1997; MATTHEWs et al., 2002; GÓMEZ and PACHeCO, 2004; FERRÁES, 2005; ABAIMOV et al., 2007, 2008), gamma (UTSU, 1984, MATthews et al., 2002; GómeZ and PACHECO, 2004; FERRÁES, 2005), power-law (SORNETTE and KNOPOFF, 1997), Brownian passage time (MATTHEWs et al., 2002; WGCEP, 2003; MiCHAEL, 2005; ABAIMOV et al., 2007; ZÖLLER et al., 2008), among others. However, due to the scarcity of registered large earthquakes in a specific fault (usually 4 to 10 earthquakes), the statistics upon which the selection of a specific pdf is based are poor. This means that different pdfs can fit the empirical distribution function.

Most of the probability distributions have been used solely for their statistical properties, 
with no relationships with the physics of the underlying process (elastic rebound theory). However, a subset of them has a physical rationale and from this point of view can be considered as better motivated. One example is the Brownian passage time distribution (BPT; MATTHEWS et al., 2002) where the seismic cycle in a fault is modelled by the time evolution of the so-called Brownian relaxation oscillator.

Also, the majority of the probability distributions used in the context of earthquake recurrence are continuous. However, in the last ten years several discrete probability distributions that are the outcome of cellular automata models have been proposed (VÁZQUEZPRADA et al., 2002; GONZÁlez et al., 2005; TEJEDOR et al., 2009). These discrete, cellular automata-based probability distributions share with the BPT distribution their physical motivation, as the models behind these discrete probability distributions try to reproduce in a few cellular automata rules the physics of a seismic fault under the elastic rebound assumption.

The aim of this paper is to present a discrete probability distribution, the negative binomial distribution (NBD) for the recurrence of large earthquakes. The study of one-way Markov cycles was presented in TEJEDOR et al. (2012), together with two of its limits, the so-called box model and the NBD. Here we focus on the NBD for its particular importance: the NBD seems to be the unique distribution that derives from the dynamics of a cellular automaton and simultaneously appears in general textbooks in probability and statistics. In Section 2 the NBD and its first moments are introduced. Section 3 recalls that the NBD is a special case of a waiting time distribution for a one-way Markov cycle, as deduced in TEJEDOR et al. (2012); Section 4 then uses this distribution as a renewal model for large earthquakes, using the earthquake series of the Parkfield segment of the San Andreas Fault as an example. The quality of the fit of the NBD to the empirical distribution function of the Parkfield series is compared to other renewal models used in the literature. Section 5 assesses the forecasting capabilities of the NBD by means of a reference prediction strategy and error diagrams. Finally, in Section 6 the most important conclusions drawn from the paper are stated. The computation of the asymptotic limit of the hazard rate for the NBD is detailed in the Appendix. 


\section{The Negative Binomial Distribution}

As there are some different modalities of defining the NBD, we will specify now the form used in this paper.

A negative binomial experiment is a statistical experiment that has the following properties: The experiment consists of $\mathrm{n}$ repeated trials. Each trial can result in just two outcomes, a success or a failure. The probability of success, denoted by $1-a(a<1)$, is the same on every trial. In consequence, the probability of failure is $a$. The trials are independent. And the experiment continues until $\mathrm{N}$ successes are observed. $N$ is specified in advance.

The negative binomial random variable is the number $n$ of repeated trials to produce $N$ successes in a negative binomial experiment. The probability distribution of the negative binomial random variable is called a NBD. Its form is:

$$
P_{N, a}(n)=(1-a)^{N} a^{n-N}\left(\begin{array}{c}
n-1 \\
N-1
\end{array}\right)
$$

The mean, variance, and coefficient of variation - or aperiodicity- of this distribution are:

$$
\begin{gathered}
\mu=\frac{N}{1-a}, \\
\sigma^{2}=\frac{N a}{(1-a)^{2}},
\end{gathered}
$$

and

$$
\alpha \equiv \frac{\sigma}{\mu}=\sqrt{\frac{a}{N}}
$$

respectively.

\section{The NBD as the waiting time distribution in an specific}

\section{Markov cycle model}

Let suppose a Markov chain with $N$ sites forming a closed loop which is gone over clockwise.

See Figure 8 for illustration. The $N$ sites are ordered by the index $i, i=0,1, . ., N-1, N$. As a genuine 
cellular automaton, time increases in discrete steps. At the beginning of each cycle our system occupies the first position, $i=0$. In the first time step it makes a trial to pass to site $i=1$. The probability of success is $\left(1-a_{0}\right)$ and that of failure $a_{0}$. Typically, after some trials the system will occupy site 1 . Now all is identical to the first case except that the probability of passing from site 1 to site 2 is $\left(1-a_{1}\right)$. Then is the turn of sites $2,3, \ldots, N-1$.

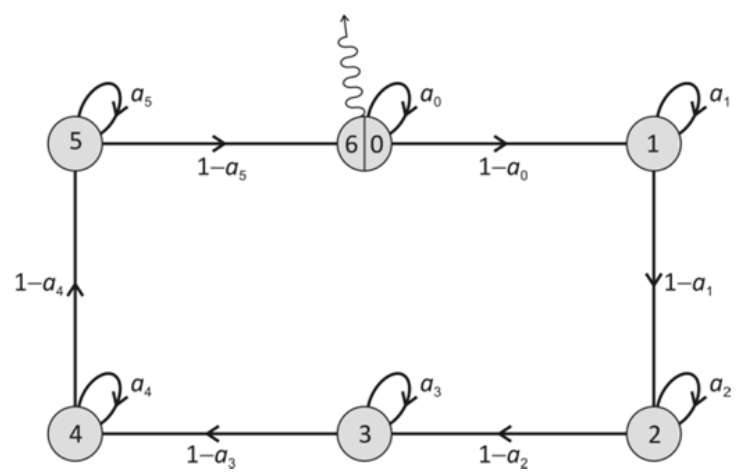

Figure 1. Scheme of a one-way Markov cycle with $\mathrm{N}=6$. The probability of staying in state $\mathrm{i}$ is $\mathrm{a}_{\mathrm{i}}$ and the probability of jumping from state $\mathrm{i}$ to state $\mathrm{i}+1$ is $\left(1-\mathrm{a}_{\mathrm{i}}\right)$. Jumping from one state to the next means that the fault has accumulated more strain energy. The wavy line between states 6 and 0 indicates that at the end of the cycle all the stored energy is released.

When site $N$ is occupied, the cycle ends. The system automatically passes to site 0 and a new cycle starts. Figure 9 shows an example of this process of slow filling and abrupt emptying for eleven consecutive cycles for a system with $N=6$ and $\left(1-a_{i}\right)=1 / N=1 / 6$, for all states $i$.

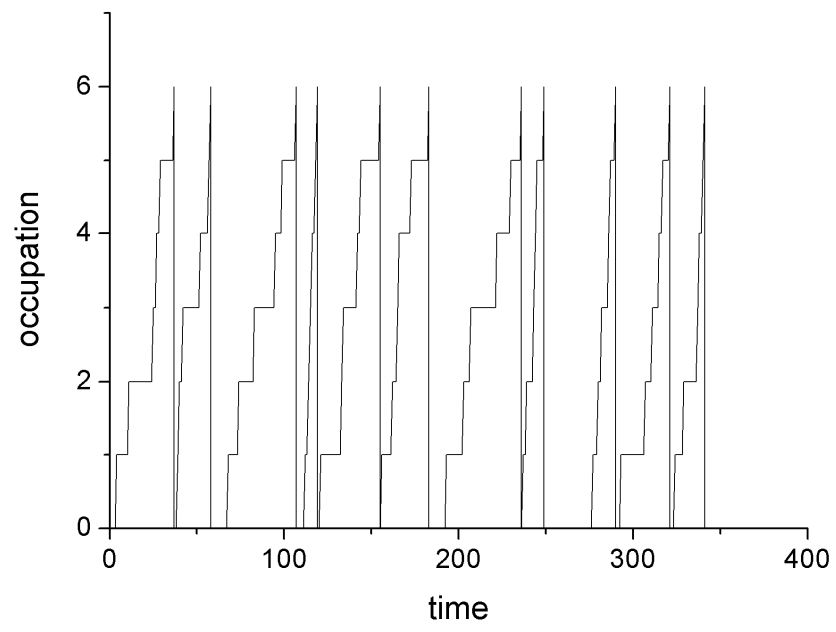

Figure 2. Occupation (number of occupied sites) of an $N=6$ system as a function of time for eleven consecutive cycles. Note the repetitive pattern but the lack of perfect periodicity. 
The travelling in successive discrete steps around the cycle can be interpreted as a process of gradual increase of strain in fault and thus this Markov cycle represents the seismic cycle in a fault. Site 0 represents the state with no strain and site $N$ represents the state of maximum strain which is automatically released to pass to site 0 . This sudden release of strain simulates the occurrence of a characteristic earthquake in the fault. Thus in this model a decrease in the strain, such as could take place in a random walk type model, is forbidden. This model is illustrated in Figure 8 and materialized in the following Markov matrix:

$$
[M]=\left(\begin{array}{cccccc}
a_{0} & 1-a_{0} & 0 & 0 & 0 & 0 \\
0 & a_{1} & 1-a_{1} & 0 & 0 & 0 \\
0 & 0 & a_{2} & 1-a_{2} & 0 & 0 \\
0 & 0 & 0 & a_{3} & 1-a_{3} & 0 \\
0 & 0 & 0 & 0 & a_{4} & 1-a_{4} \\
1-a_{5} & 0 & 0 & 0 & 0 & a_{5}
\end{array}\right)
$$

Note that the number of parameters in this discrete model is $N+1$ : the length of the cycle, $N$, plus the value of the $N$ parameters $a_{i}$. Using standard techniques of Markov chains (TEJEDOR et al, 2012) one can obtain, in a closed form, the distribution function of the cycle lengths in this model:

$$
P_{N}(n)=\prod_{i=0}^{N-1}\left(1-a_{i}\right) \sum_{i=0}^{N-1}\left[\frac{a_{i}^{n-1}}{\prod_{j(\neq i)=0}^{N-1}\left(a_{i}-a_{j}\right)}\right], \quad n=N, N+1, \ldots, \infty
$$

It is clear that until time step $n=N$ the probability of completing a cycle is null. In seismology this is called a stress-shadow..

A property of this general model is that no matter what the value of its parameters are, the aperiodicity is lower than 1 .

When the $N$ parameters $a_{i}$ are equal,

$$
a=a_{1}=a_{2}=\ldots . .=a_{N}
$$

Eq. (7) becomes Eq. (2). That is, if Eq. (8) is fulfilled a NBD is the waiting time distribution of the Markov cycle.

After this hypothesis, the pdf has only two parameters $N$ and $a$. This bi-parametric freedom can be used for fitting purposes, including of course the seismic cycles. In this paper, however, 
we will step forward with an additional simplification by relating them in the form:

$$
1-a=\frac{1}{N}
$$

After this new hypothesis, there is only one free parameter and each cycle of the model can be intuitively associated with the ordered filling of a box with $N$ positions. The new simplified NBD is

$$
P_{N}(n)=\left(\frac{1}{N}\right)^{N}\left(\frac{N-1}{N}\right)^{n-N}\left(\begin{array}{c}
n-1 \\
N-1
\end{array}\right), \quad n=N, N+1, \ldots \infty
$$

In the next section, we will see that $N=6$ is the most appropriate size of the model to fit the recurrence of earthquakes in the Parkfield, California, section of the San Andreas Fault. For this case the pdf in Eq. (10) is simply:

$$
P_{6}(n)=\left(\frac{1}{6}\right)^{6}\left(\frac{5}{6}\right)^{n-6}\left(\begin{array}{c}
n-1 \\
5
\end{array}\right), \quad n=6,7, \ldots \infty
$$

And the values of its mean ad aperiodicity are:

$$
\mu_{6}=36 \text { and } \alpha_{6}=0.373
$$

Figure 10 plots the NBD written in Eq. (11). To remark the discrete nature of the probability distribution only points for integer time steps have been drawn, with no line connecting them.

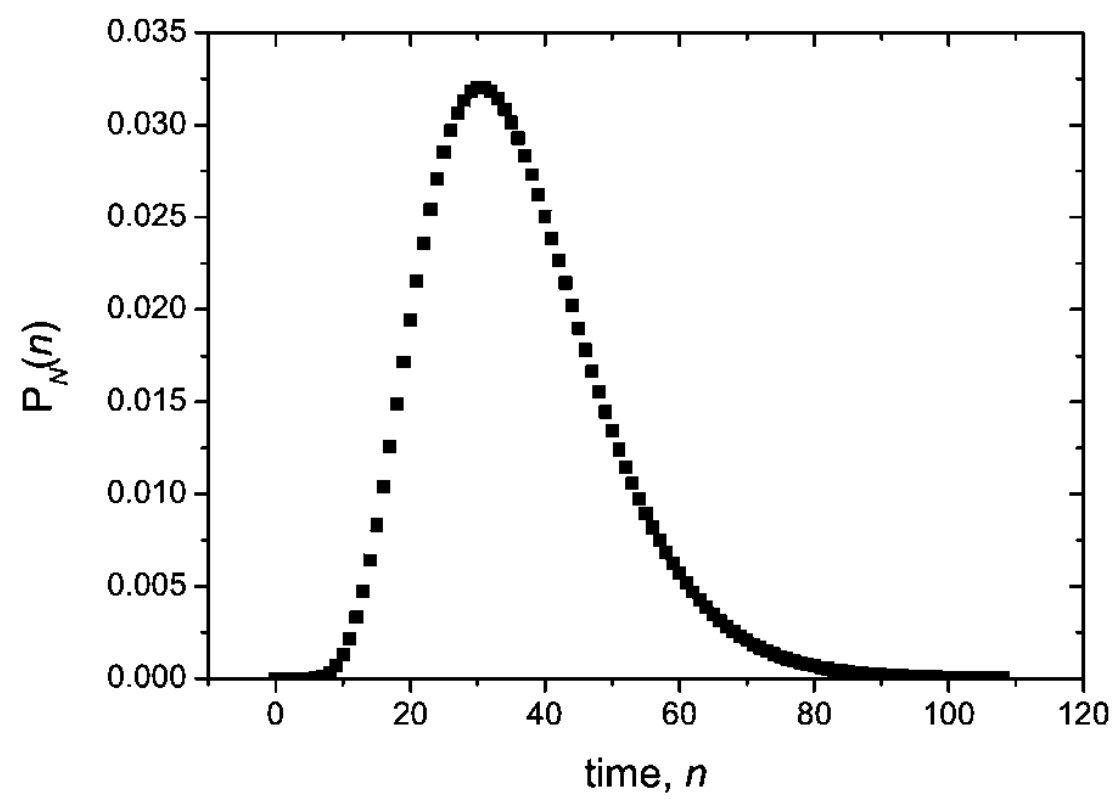

Figure 3. Probability density function of the NBD for the case $\mathrm{N}=6,(1-\mathrm{a})=1 / 6$. 


\section{Applications of the NBD in seismicity and earthquake}

\section{forecasting: the Parkfield series}

Including the latest event, the Parkfield series (BAKUN and LINDH, 1985; BAKUN, 1988; MiCHAEL and JONES, 1998) consists of seven $M_{\mathrm{w}} \approx 6$ mainshocks, which occurred on January 9 , 1857; February 2, 1881; March 3, 1901; March 10, 1922; June 8, 1934; June 28, 1966 and September 28, 2004. In consequence, the duration (in years) of the six observed inter-event times are: $24.07,20.08,21.02,12.25,32.05$ and 38.25 . The mean value $\mu_{\mathrm{Pk}}$, the sample standard deviation $\sigma_{\mathrm{Pk}}$ (the square root of the bias-corrected sample variance), and the aperiodicity $\alpha_{\mathrm{Pk}}$ of this six-data series are:

$$
\mu_{\mathrm{Pk}}=24.62 \mathrm{yr} ; \sigma_{\mathrm{Pk}}=9.25 \mathrm{yr} ; \alpha_{\mathrm{Pk}}=0.3759
$$

Now, we will proceed to fit these data using the simplified NBD written in Eq (11). Its aperiodicity is given by

$$
\alpha_{\mathrm{NBD}}=\sqrt{\frac{N-1}{N^{2}}}
$$

As we want a distribution with the same aperiodicity (and mean) as the Parkfield series, taking $\alpha_{\mathrm{Pk}}$ from Eq. (13) and substituting it in Eq. (14), we have $N=5.8$. But because $N$ is a discrete quantity, we use the nearest integer, $N=6$.

However, for fitting the data, it is necessary to assign a definite number of years to the nondimensional time step of the model. This second parameter will be called $\tau$. From Eqs. (3)and (9) we have that for the NBD $\mu=N^{2}=36$ time steps. This mean cycle length (in nondimensional time steps) should be equal to the mean recurrence time of the Parkfield series, $\mu_{\mathrm{Pk}}$ $=24.62$ years, so that $\tau=0.68$ years per time step of the model. In Figure 11 we have plotted the empirical distribution function of the Parkfield series (gray step-like line) and the fit to the cumulative NBD with $N=6$ (black continuous line), together with 5 other (cumulative) distribution functions used as renewal models in the literature: Weibull, gamma, log-normal, BPT and Minimalist Model (MM; VAZQUEZ-PRADA et al., 2002). It is quite obvious from the 
figure that the performance of all six models is good and very similar, including the NBD. Indeed, the residuals for the NBD evaluated at the midpoints of the horizontal segments of the empirical distribution function are the lowest of the six tested models.

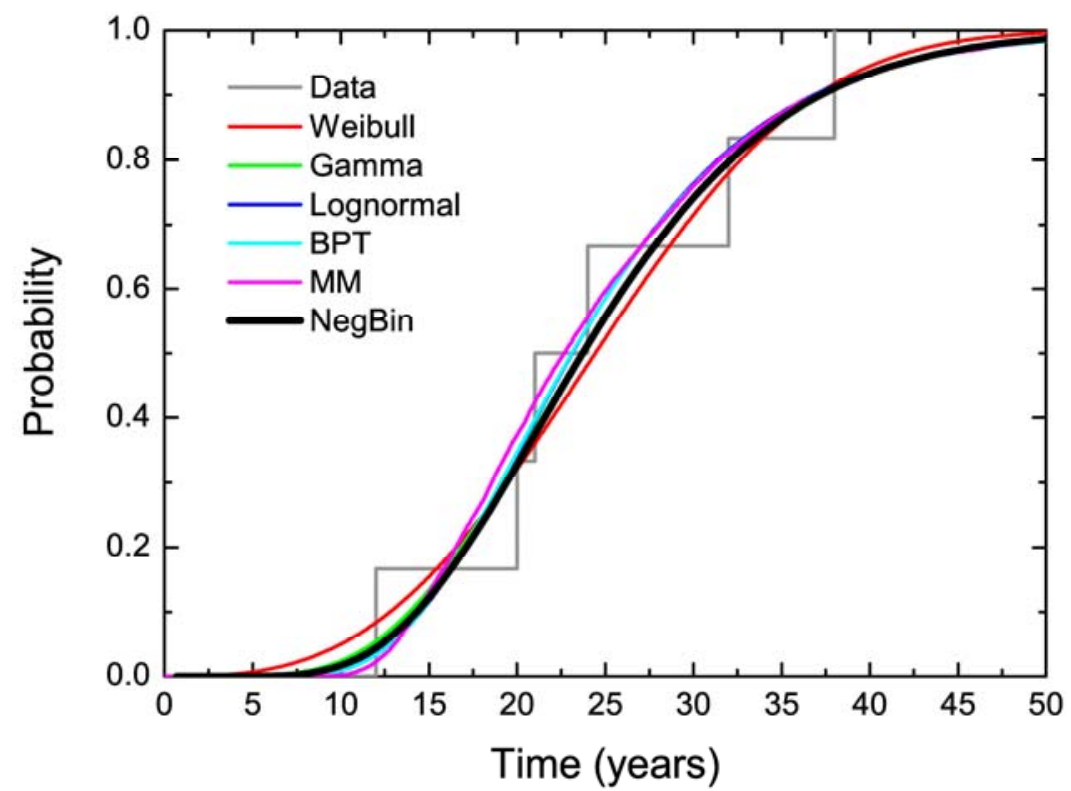

Figure 4. Fit of the NBD model (black continuous line) to the Parkfield series (gray step-like line) and comparison with other statistical models used in the literature.

The NBD (and any of the other models shown in Figure 11) can be used to estimate the time-dependent probability of having an earthquake as a function of the time elapsed since the last earthquake in the series (September 28, 2004). This estimation can be carried out with the hazard rate function,

$$
h_{N, a}(n)=\frac{P_{N, a}(n)}{\sum_{i=n}^{\infty} P_{N, a}(i)}
$$

For discrete distributions like the NBD, the hazard rate is the probability for an earthquake to occur at time step $n$ on the condition that it has not occurred until time step $n-1$. However, in the seismological literature is customary to express the likelihood of a future earthquake using the yearly conditional probability of earthquake occurrence, $P(n \mid \Delta t=1$ year), instead of the hazard rate. This function gives the probability of having an earthquake during the next year provided it has not occurred before: 


$$
P_{N, a}(n \mid \Delta t=1 \text { year })=\frac{S_{N, a}(n+\Delta t)-S_{N, a}(n)}{1-S_{N, a}(n-1)}
$$

where $S_{N, a}(n)=\sum_{n^{\prime}=N}^{n} P_{N, a}\left(n^{\prime}\right)$ is the cumulative distribution function. The yearly conditional probability function for the Parkfield series is illustrated in Figure 12. Again, as in Figure 11, the NBD and five other models are compared. The present yearly probability of earthquake occurrence is 0.004 , i.e., there is a $0.4 \%$ probability of having an earthquake in the following 12 months. Obviously this probability is low because the earthquake cycle is in its early stages. When the cycle is at its average duration, 24.62 years, the yearly probability of earthquake occurrence will be $6 \%$.

Both the hazard rate and the yearly conditional probability functions for the NBD reach a constant value for large times. Inserting Eq. (2)) into Eq. (15) one obtains that, for long times,

$$
\lim _{n \rightarrow \infty} h_{N, a}(n)=1-a
$$

The derivation of this equation can be found in the Appendix. If Eq. (9) is used instead (i.e., the one-parameter simplification of the NBD), the asymptotic limit of the hazard function is equal to $1 / N$.

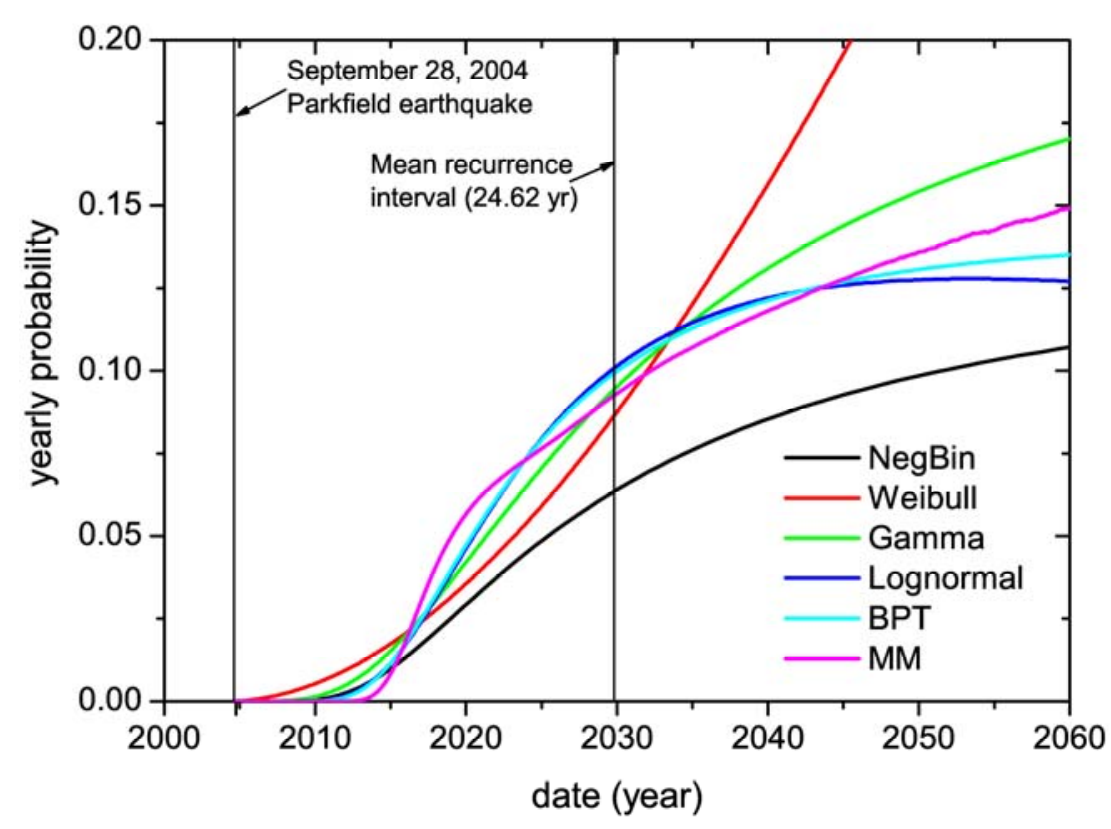

Figure 5. Yearly conditional probability for the Parkfield series as predicted by the Negative Binomial model compared to other statistical models used in the literature. 


\section{Error diagrams for the Parkfield example}

A hint of the predictability of the large relaxations in this type of model is given by the aperiodicity of their time series. The aperiodicity, as stated in Section 1, is a quantitative measure of the lack of regularity of a time series. As the aperiodicity of this model is always less than 1 , the occurrence of the large events is a quasi-periodic phenomenon. A robust way to assess the predictability of a time series is by trying to forecast its events by declaring alarms at particular times.

The aim is to declare alarms before all the events in order not to miss any, but to declare them just before each event in order to minimize the total alarm time. Many strategies can be devised to declare the alarms but there is a reference strategy to which all others can be compared (NEWMAN and TURCOTTE, 1992; VÁZQUEZ-PRADA et al., 2002; KEILIS-BOROK and SOLOVIEV, 2003). This strategy consists of waiting a fixed time after each event (waiting time w), setting then the alarm, and maintaining it until the occurrence of the next event (Figure 13). If the following event in the time series occurs before the alarm is raised, it is counted as a prediction error; if the following event in the time series occurs after the alarm is raised, it is counted as a prediction success and the alarm is then cancelled.

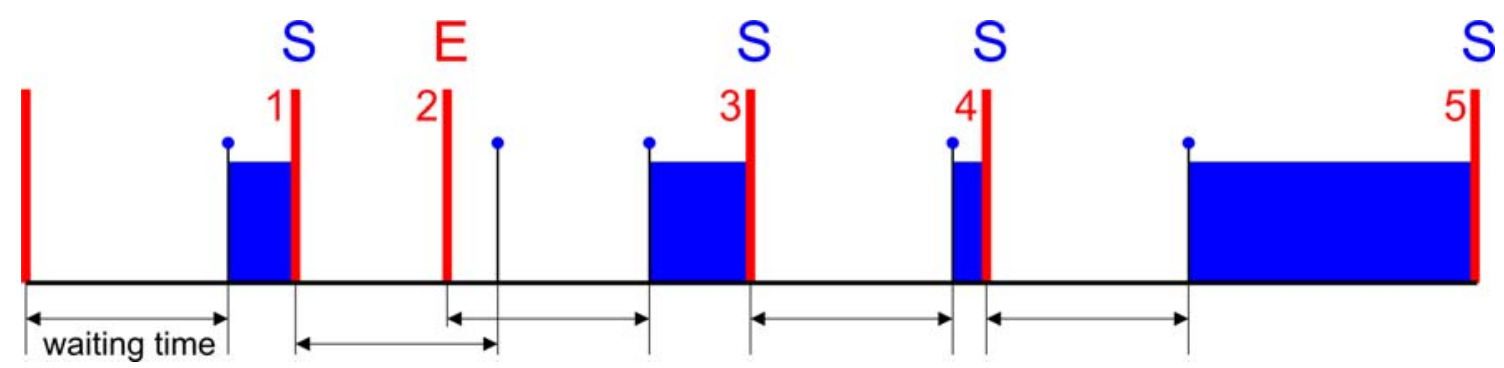

Figure 6. Reference strategy for the assessment of the predictability of a time series. Red bars are the earthquakes to be forecasted (five in the example). An S (success) above a red bar means that the earthquake has been successfully predicted, whereas an E (error) means that the earthquake has not been predicted. The blue strips stand for the time with the alarm on before each earthquake.

The events that are to be predicted (large earthquakes) are the vertical red bars numbered 
correlatively. An alarm (vertical black lines with rounded top) is set a fixed time interval after each event (waiting time) and the prediction is labelled error (E) or success (S) depending on whether the alarm was off or on when the event occurred, respectively. The fraction of errors is the number of events not predicted (one in the example, the second event) divided by the total number of events (five events), i.e., $f_{e}=0.2$; and the fraction of alarm time is the total alarm time (blue sections of the time line: 29 time units) divided by the total duration of the time series (86 time units), i.e., $f_{a}=0.34$ in the example shown in the figure.

The fraction of errors $f_{e}$ (number of missed events divided by the total number of events) and the fraction of alarm time $f_{a}$ (total alarm time divided by the total duration of the time series) can be computed as a function of the above mentioned waiting time $w$, and the purpose is to find the optimum waiting time. This optimum waiting time depends on the relative importance that failing to predict an event has compared to keeping the alarm on. An objective function, called loss function, $L$, can be defined that incorporates this trade-off in each particular case. Here we will use the simplest of them, $\mathrm{L}=f_{e}+f_{a}$, where failure to predict an event and a longer alarm time are equally penalized.

Thus, the aim is to find the waiting time $w=w^{*}$ that minimizes $L(w)$. This minimum value is denoted by $L^{*} \equiv L\left(w^{*}\right)$. And the best way to graphically display this is by means of an error diagram, where the fraction of alarm time $f_{a}$ runs along the horizontal axis and the fraction of errors $f_{e}$ runs along the vertical axis. Error diagrams were introduced in earthquake forecasting by MOLCHAN (1997) who contributed with rigorous mathematical analysis to the optimization of the earthquake prediction strategies.

A good strategy of forecasting must produce both small $f_{e}$ and $f_{a}$, because both the prediction failures and the alarms are costly. A random guessing strategy (randomly turning the alarm on and off) will yield $L=1$, a result which can be easily understood. The alarm will be on, randomly, during a certain fraction of time, $f_{a}$. Thus, there will be a probability equal to fa for it being on when an earthquake eventually occurs (and a probability of $1-f_{a}$ for it being off). The result is that $\mathrm{fe}=1-f_{a}$. As a trivial special case, if the alarm is always on $\left(f_{a}=1\right)$, then all the earthquakes are "forecasted" $\left(f_{e}=0\right)$. Conversely, all the earthquakes are failures to predict if the 
alarm is always off. The random guessing strategy is considered as a baseline, so a forecasting procedure makes sense only if it gives $f_{a}+f_{e}<1$.

Both functions, $f_{a}$ and $f_{e}$, together with the loss funtion $L=f_{e}+f_{a}$ are plotted in Figure 14a for the case $N=6$, while Figure $14 \mathrm{~b}$ plots the error diagram for the same data. For each value of $N$, $L(w)$ has a minimum at a specific value of $w, w^{*}(N)$. As can be seen in Figure 14, $w^{*}(6)=22$, for which

$$
f_{a}\left(w^{*}\right)=0.403, f_{e}\left(w^{*}\right)=0.147, L\left(w^{*}\right)=0.550
$$
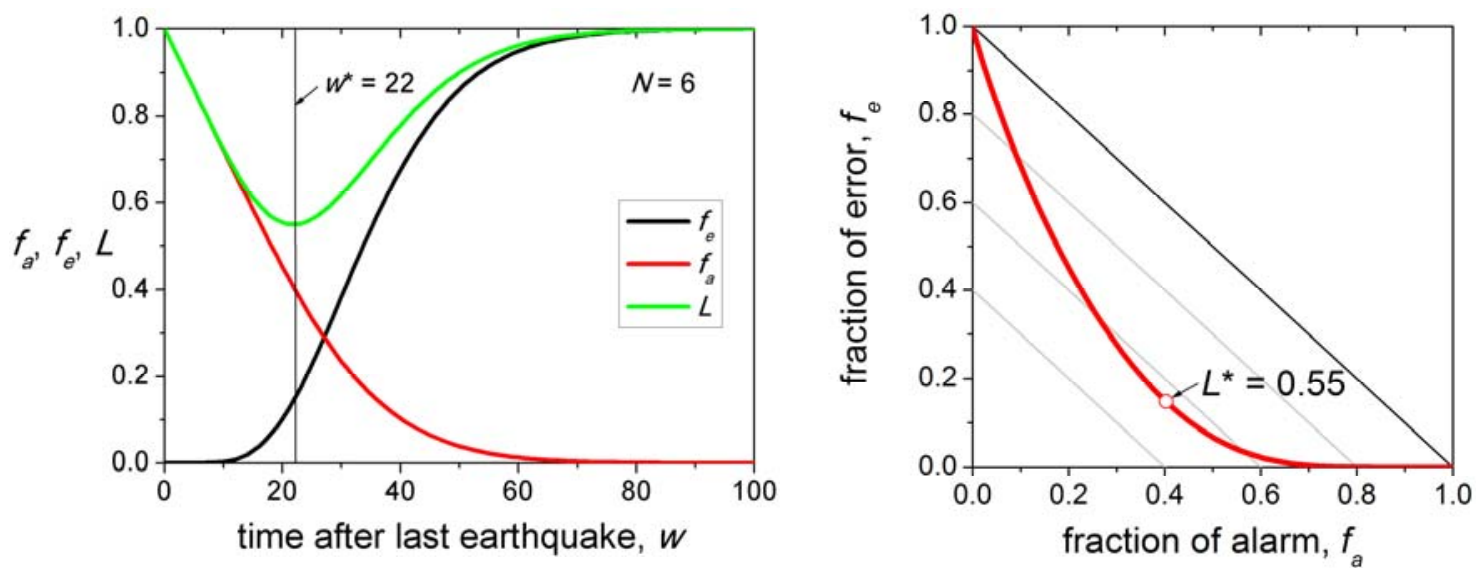

Figure 7. (a) Fraction of error $\mathrm{f}_{\mathrm{e}}$, fraction of alarm, $\mathrm{f}_{\mathrm{a}}$ and loss function $\mathrm{L}$ as a function of the time after the last earthquake for a NBD model with $\mathrm{N}=6$. (b) Error diagram for the prediction strategy shown in (a). The minimum value of the loss function is $\mathrm{L}^{*}=0.55$ for $\mathrm{w}^{*}=22$.

For the Parkfield sequence, $w^{*}$ corresponds to $\tau w^{*}=15.0 \mathrm{yr}$

If the distribution derived from the NBD model correctly describes the recurrence of large earthquakes at Parkfield, an alarm connected 15 years after the last earthquake (beginning of the cycle) and disconnected just after the occurrence of each shock would yield the results given in Eq. (18). Note that this time is approximately equal to the difference between the mean and the standard deviation. This is reasonable because $w^{*}=15 \mathrm{yr}$ would capture most of the probability curve, as can be seen in Fig. 3. 


\section{Conclusions}

We have introduced the NBD as a renewal model to describe the recurrence of large earthquakes in faults.

As a test ground of application, we have used the Parkfield series. The yearly conditional probability and other functions as predicted by the NBD are compared to other statistical models used in the literature, and a simple forecasting strategy has been evaluated using error diagrams.

Our results show that the NBD is competitive against other models but general conclusions cannot be drawn because of the smallness of the sample.

The NBD seems to be the unique discrete distribution coming from a cellular automaton whose properties can be found in textbooks of probability and statistics.

In this paper we have reduced one parameter of the distribution by relating the probability of advancing in the Markov process to the total number of steps in the cyclic chain. With this simplification, this model can be intuitively understood as the progressive ordered filling of a finite box.

\section{Appendix A: Asymptotic behavior of the hazard rate function}

Recall that the $N$-step Markov-cycle distribution, Eq. (7), collapses to a negative binomial distribution when all transition probabilities are equal, $a=a_{1}=a_{2}=\ldots \ldots=a_{N}$ :

$$
P_{N, a}(n)=(1-a)^{N} a^{n-N}\left(\begin{array}{c}
n-1 \\
N-1
\end{array}\right)=\left(\frac{1-a}{a}\right)^{N} a^{n} \frac{(n-1) \ldots . .(n-N+1)}{(N-1) !} .
$$

Using the definition of hazard rate for a discrete distribution, Eq. (15) we can write

$$
h_{N, a}(n)=\frac{P_{N, a}(n)}{\sum_{i=n}^{\infty} P_{N, a}(i)}=\frac{a^{n}(n-1) \ldots(n-N+1)}{\sum_{i=n}^{\infty} a^{i}(i-1) \ldots . .(i-N+1)}=\frac{1}{\sum_{i=1}^{\infty} a^{i-n} \frac{i-1}{n-1} \ldots \frac{i-N+1}{n-N+1}} .
$$

To proceed further, we make the following change of variable:

$$
i-n=m \text {. }
$$


With this change of variable the hazard rate of the general, two-parameter NBD, Eq. ( .19), can be written as

$$
h_{N, a}^{-1}=\sum_{m=0}^{\infty} a^{m}\left(1+\frac{m}{n-1}\right) \ldots . .\left(1+\frac{m}{n-N+1}\right) .
$$

In the long-time limit, i.e., when $n$ tends to infinity, we have

$$
\lim _{n \rightarrow \infty} h_{N, a}^{-1}=\sum_{m=0}^{\infty} a^{m}(1 \times 1 \times 1 \ldots \times 1)=\sum_{m=0}^{\infty} a^{m}=\frac{1}{1-a} .
$$

So, in the general, two-parameter NBD the asymptotic limit of the hazard rate is:

$$
\lim _{n \rightarrow \infty} h_{N, a}=1-a \text {. }
$$

\section{REFERENCES}

Abaimov, S.G., TuRCotTe, D.L. and RundLe, J.B. (2007), Recurrence-time and frequency-slip statistics of slip events on the creeping section of the San Andreas fault in central California, Geophys. J. Int. 170, 1289-1299.

Abaimov, S.G., Turcotte, D.L., Shcherbakov, R, Rundle, J.B. YAKovlev, G., GoltZ, C., and Newman, W.I. (2008), Earthquakes: Recurrence and Interoccurrence Times, Pure appl. geophys. 165, 777-795.

BAKUN, W.H. (1988), History of significant earthquakes in the Parkfield area, Earthq. Volcano. $20,45-51$.

BAKUn, W.H., and LindH, A.G. (1985), The Parkfield, California, earthquake prediction experiment, Science 229, 619-624.

Ellsworth, W.L., Matthews, M.V., NAdeau, R.M., Nishenko, S.P., ReasenberG, P.A., SIMPSON, R.W. (1999), A physically-based earthquake recurrence model for estimation of long-term earthquake earthquake probabilities. United States Geological Survey Open-File Report 99, 552pp.

FERRÁES, S. (2003), The conditional probability of earthquake occurrence and the next large earthquake in Tokyo, Japan, J. Seismol. 7, 145-153. 
FERRÁES, S. (2005), A probabilistic prediction of the next strong earthquake in the AcapulcoSan Marcos segment, Mexico, Geofísica Internacional 44(4), 347-353.

GómeZ, J.B. and PACHECO, A.F. (2004), The Minimalist Model of characteristic earthquakes as a useful tool for description of the recurrence of large earthquakes, Bull. Seismol. Soc. Am. 94, 1960-1967.

GONZÁLEZ, Á., GÓMEZ, J.B. and PACHECO, A.F. (2005), The occupation of a box as a toy model for the seismic cycle of a fault, Am. J. Phys. 73, 946-952.

KeILIS-Bork D. V. and SolovieV A. (2003), Nonlinear Dynamics of the Lithosphere and Earthquake Prediction, Springer Verlag, Berlin.

Matthews, M.V., Ellsworth, W.L. and Reasenberg, P.A. (2002), A Brownian model for recurrent earthquakes, Bull. Seismol. Soc. Am. 92, 2233-2250.

Michael, A.J. and Jones, L.M. (1998), Seismicity alert probabilities at Parkfield, California, revisited, Bull. Seismol. Soc. Am. 88, 117-130.

MiCHAEL, A.J. (2005), Viscoelasticity, postseismic slip, fault interactions, and the recurrence of large earthquakes, Bull. Seismol. Soc. Am. 95, 1594-1603.

Molchan, G.M. (1997), Earthquake prediction as a decision-making problem, Pure Appl. Geophys. 149(1), 233-247.

NEWMAN W. I. and TURCOTTE D.L. (1992), A simple model for the earthquake cycle combining self-organized complexity with critical point behavior, Nonlinear Process. Geophys. 9, $453-61$.

REID, H.F. (1910), The mechanics of the earthquake, In: The California Earthquake of April 18, 1906, Report of the State Earthquake Investigation Commission, Carnegie Institution, Washington, DC, Vol. 2, pp. 1-192.

RIKITAKE, T. (1974), Probability of earthquake occurrence as estimated from crustal strain, Tectonophysics 23(3), 299-312.

ScHOLZ, C.H. (2002), The Mechanics of Earthquakes and Faulting, Cambridge University 
Press.

SornetTe, D. and KnOpoff, L. (1997). The paradox of the expected time until the next earthquake. Bull. Seismol. Soc. Am. 87, 789-798.

SYKES, L.R., and MENKE, W. (2006), Repeat Times of Large Earthquakes: Implications for Earthquake Mechanics and Long-Term Prediction. Bull. Seismol. Soc. Am. 96(5), $1569-1596$.

TEJEDOR, A., GÓMEZ, J.B., and PACHECO, A.F. (2009), Earthquake size-frequency statistics in a forest-fire model of individual faults, Physical Review E 79, 046102.

TEJedOR, A., GóMEZ, J.B., and PACHECO, A.F. (2012), One-way Markov process approach to repeat times of large earthquakes in faults, J. Stat. Phys. 149(5), 951-963.

UTSU, T. (1984), Estimation of parameters for recurrence models of earthquakes, Bull. Earthq. Res. Inst. Univ. Tokyo 59, 53-66.

VÁzQuez-Prada, M., GonzÁlez, Á., Gómez, J.B. and Pacheco, A.F. (2002), A minimalist model of characteristic earthquakes. Nonlinear. Process. Geophys. 9, 513-519.

Working Group on CALIFornia EARTHQuake Probabilities (2003), Earthquake Probabilities in the San Francisco Bay Region: 2002-2031, United States Geological Survey Open-File Report 03-214, 234 p.

Yakovlev, G., Turcotte, D.L., Rundle, J.B., and Rundle, P.B. (2006) Bull. Seismol. Soc. Am 96(6), 1995-2007.

ZÖLleR, G., HAINZL, S., and HOLSCHNEIDER, M. (2008), Recurrent Large Earthquakes in a Fault Region: What Can Be Inferred from Small and Intermediate Events?, Bull. Seismol. Soc. Am. 98, 2641-2651. 